Article original

\title{
Profil épidémiologique du cancer du sein dans l'Ouest Algérien
}

\author{
Epidemiological profile of breast cancer in Western Algeria
}

\author{
Nadia Bessaih', Leila Houti² \\ ${ }^{1}$ Service d'Oncologie, EHU Oran. Faculté de Médecine d'Oran , Algérie. \\ ${ }^{2}$ LABSIS. Faculté de Médecine d'Oran
}

\section{MOTS CLÉS \\ Cancer du sein; ménarchie précoce; nulliparité; obésité; facteurs de risque modifiables.}

'Auteur correspondant : bessaih.nadia@univ-oran.dz

\section{Résumé} de vie.
Introduction - Le cancer du sein constitue un problème de santé publique en Algérie, et il est important aujourd'hui d'identifier les groupes à risque afin d'optimiser les programmes de dépistage. L'objectif de cette étude est de dresser le profil épidémiologique du cancer du sein dans l'Ouest Algérien et d'identifier ses facteurs de risque en les appréciant selon les groupes d'âge, les données anthropométriques et le mode

Matériels et méthodes - II s'agit d'une étude cas-témoins (210 cas et 265 témoins recrutés dans une consultation de médecine générale et appariés sur l'âge et le niveau socio-économique) menée au service d'Oncologie du CHU d'Oran. Les facteurs de risque abordés dans le questionnaire sont la ménarchie précoce, la parité, la prise de contraceptifs oraux, l'âge à la première grossesse, l'allaitement, la ménopause tardive et l'obésité.

Résultats - Les résultats montrent que la nulliparité constitue un facteur de risque avant 40 ans $(O R=14,4, p<0,001)$ et entre 40 et 69 ans $(O R=12,93, p<0,002)$. La ménarchie précoce intervient dans le risque chez les 40-69 ans ( $O R=5,44, p=0,001)$. Chez les femmes de plus de 70 ans, l'obésité apparait comme un facteur de risque $(\mathrm{OR}=1,48, \mathrm{p}<0,001)$. L'association du cancer et de la prise de contraceptifs n'a pas été retrouvée dans cette série.

Conclusion - La ménarchie précoce, la nulliparité et l'obésité sont les facteurs de risque du cancer du sein chez la femme dans l'Ouest Algérien La prévention du cancer du sein réside dans l'action sur les facteurs de risque modifiables tels que l'obésité. Chez les femmes de moins de 40 ans, il est recommandé d'individualiser le dépistage par des tests génétiques systématiques. 


\section{KEY WORDS}

Breast cancer; early menarche; nulliparity; obesity; modifiable risk factors.

\begin{abstract}
Introduction - Breast cancer is a public health problem in Algeria, and it is important today to identify the risk groups in order to optimize screening programs. The objective of this study is to discribe the epidemiological profile of breast cancer in West Algeria and to identify its risk factors according to age groups, anthropometric data and lifestyle.
\end{abstract}

Materials and methods - A case-control study (210 cases and 265 witnesses recruited in a general medicine consultation and matched on age and socio-economic level) has been held at the Oncology Department at Oran Hospital. The risk factors addressed in the questionnaire are: early menstruation, parity, oral contraceptives, age at first pregnancy, breastfeeding, late menopause and obesity.

Results - The results show that nulliparity is a risk factor for 40 years $(O R=14.4$, $p<0.001)$ and between 40 and 69 years $(O R=12.93, p<0.002)$. Early menstruation occurs in risk in $40-69$ years $(O R=5.44, p=0.001)$. In women over 70 years old, obesity appears to be a risk factor $(O R=1.48, p<0.001)$. The association of cancer and oral contraceptives has not been found in this series.

Conclusion - Early menarche, nulliparity and obesity are the main risk factors for breast cancer in women in Western Algeria. Its prevention lies in the action on the modifiable risk factors such as obesity. Among women under 40 years of age, it is recommended to individualize screening by systematic genetic testing.

\section{Introduction}

Le cancer du sein est le premier cancer chez la femme et demeure un problème de santé publique dans le monde [1]. Il représente $16 \%$ des cancers féminins et constitue la cause la plus fréquente de décès par cancer chez les femmes avec 522000 décès enregistrés en 2004 [2]. L'effectif de 1,7 million de cas, est en forte hausse dans le monde entier [1]. Bien que l'on considère cette maladie comme une maladie du monde développé, d'énormes inégalités existent entre les pays riches et les pays en développement où la majorité des cas sont diagnostiqués à des stades avancés et où surviennent $69 \%$ des décès $[2,3]$.

En Algérie, le cancer du sein est en progression et son incidence est estimée à 11000 nouveaux cas par an [4]. Il touche la femme jeune. Son incidence augmente rapidement à partir de 15 ans et atteint deux pics entre 45-49 ans et 60-64 ans avec un âge médian au diagnostic qui se situe à 47 ans [5].

Sa croissance dans les pays en voie de développement est liée à l'allongement de l'espérance de vie et à la modernisation des modes de vie [6]. En Algérie, le cancer du sein survient dans un contexte de transition épidémiologique, en cours depuis quatre décennies, qui s'est accompagnée d'une modification du mode de vie collectif et individuel [7].

Plusieurs facteurs de risque ont été incriminés dans la survenue du cancer du sein [8]. Le tabagisme [9], l'obésité $[10,11]$ et la sédentarité [12], sont les plus largement incriminés. Toutefois, pour la majorité des femmes présentant un cancer du sein, il n'est pas possible d'identifier des facteurs de risque particuliers [13, 14].

Parmi les 69 facteurs de risque recensés dans la littérature scientifique, 7 méritent un dépistage spécifique [15]. Les femmes ne courent pas le même risque de cancer en général, et du cancer du sein en particulier. Les risques sont parfois liés au patrimoine génétique et peuvent augmenter au cours de l'existence en raison de choix personnels, ainsi que de facteurs liés à l'environnement [14]. Il existe encore des incertitudes quant à l'implication et au poids de ces facteurs de risque. En Algérie, la population féminine a de plus ses particularités relatives à la vie génitale, reproductive et alimentaire.

Le cancer du sein, touche autant la femme jeune que la femme âgée [1] en fonction de facteurs de risque multiples, ce qui complique son dépistage. L'identification du profil épidémiologique de cette pathologie chez la femme 
algérienne et des déterminants sur lesquels il est possible d'agir, devrait faciliter la mise en place de stratégies de prévention afin d'orienter le dépistage selon les tranches d'âge, plus particulièrement celles où les facteurs de risques sont modifiables.

L'objectif de cette étude est de dresser un profil épidémiologique du cancer du sein chez la femme dans l'Ouest Algérien et d'identifier des facteurs de risque modifiables en les appréciant selon les groupes d'âge, les données anthropométriques et le mode de vie.

\section{Matériels et méthodes}

Notre étude a été réalisée au service d'Oncologie Médicale du CHU d'Oran, sur un échantillon de 210 patientes âgées de 20 à 74 ans. Toutes les femmes se présentant pour prise en charge d'un cancer du sein avec confirmation histolo- gique, ont été admises dans l'étude. Un échantillon témoin $(n=267)$ a été recruté en parallèle, dans une consultation de médecine générale au sein d'une polyclinique de la ville d'Oran. Les témoins ont été recrutés parmi les femmes ne présentant aucune pathologie néoplasique, et appariées selon l'âge et le niveau socio-économique. Tous les sujets inclus dans ce travail sont originaires de l'Ouest Algérien.

L'enquête a été réalisée au moyen d'un questionnaire administré, qui aborde les aspects gynéco-obstétricaux et les facteurs de risque du cancer du sein. Des mesures anthropométriques associant poids et taille ont été effectuées.

Les variables étudiées sont : la tranche d'âge ( moins de 40 ans, 40-69 ans , 70 ans et plus), la ménarchie précoce avant 11 ans, la grossesse tardive après 35 ans, le délai ménarchie - âge à la première grossesse (inférieur à 12 ans, 12 ans et plus), la multiparité (plus de 5 gros-

Tableau 1 : Caractéristiques cliniques des cas et des témoins

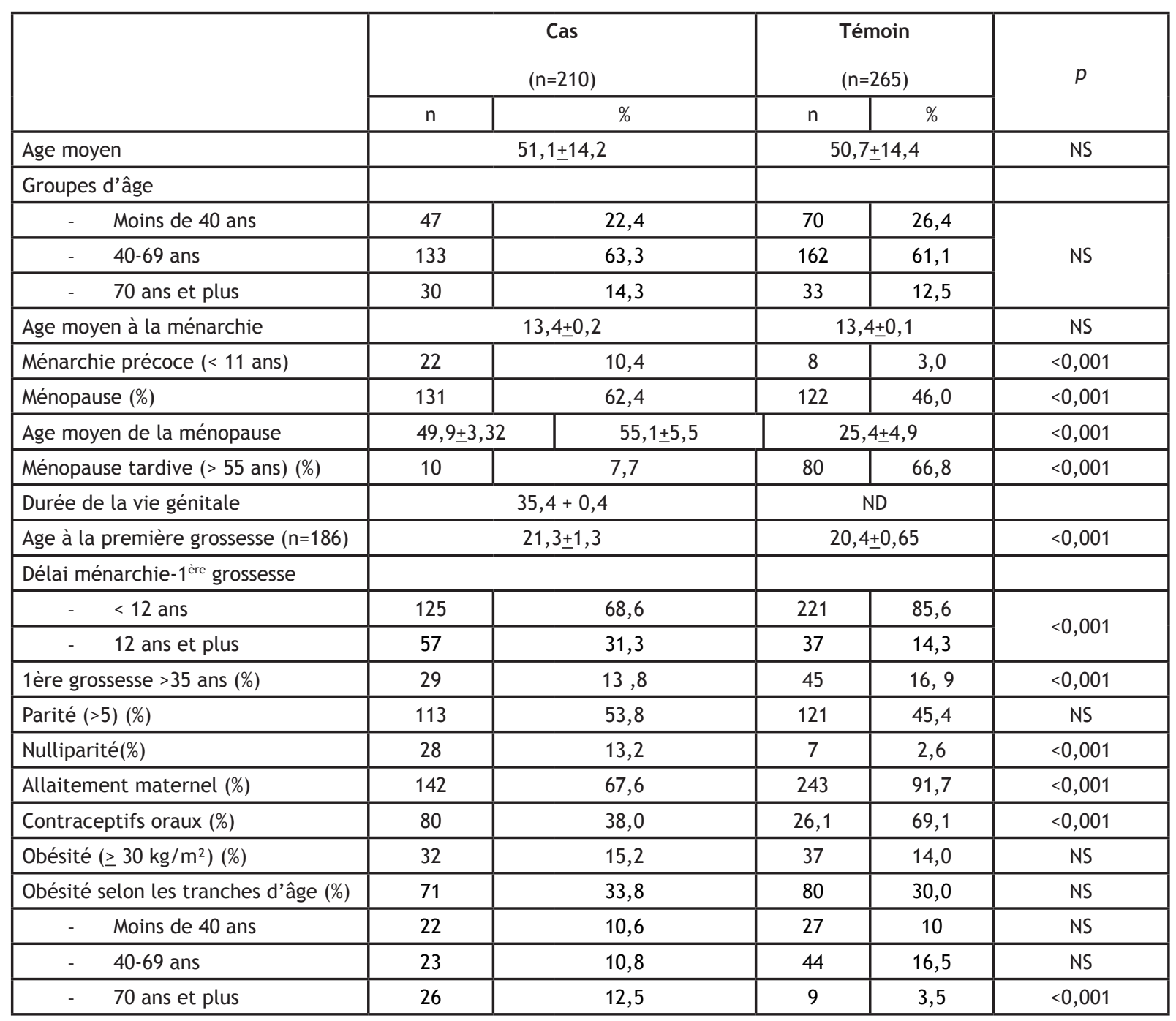


sesses), la nulliparité, l'allaitement maternel, la prise de contraceptifs, la ménopause tardive après 55 ans, la durée de la vie génitale et l'obésité $\left(I M C>30 \mathrm{~kg} / \mathrm{m}^{2}\right)$.

Le consentement éclairé a été obtenu auprès des patientes avant leur participation à l'étude, et les données ont été rendues anonymes avant leur traitement. L'analyse des données a été effectuée sur le programme Epi Info version 6 .

Tableau 2 : Analyse des facteurs de risque de cancer du sein

\begin{tabular}{|l|l|l|l|}
\hline & OR & IC à 95\% & $\mathrm{P}$ \\
\hline Age $<40$ ans & 0,99 & $0,62-1,55$ & NS \\
\hline Age $\geq 70$ ans & 0,95 & $0,53-1,71$ & NS \\
\hline Ménarchie précoce & 3,76 & $1,55-1,41$ & $<0,001$ \\
\hline Ménopause tardive & 0,12 & $0,05-0,24$ & $<0,001$ \\
\hline Nulliparité & 5,67 & $2,30-14,52$ & $<0,001$ \\
\hline Parité $>5$ & 0,31 & $0,21-0,47$ & $<0,001$ \\
\hline $\begin{array}{l}\text { Age tardif de la pre- } \\
\text { mière grossesse }\end{array}$ & 2,55 & $0,40-20,25$ & NS \\
\hline $\begin{array}{l}\text { Prise de contraceptifs } \\
\text { oraux }\end{array}$ & 0,26 & $0,18-0,41$ & $<0,001$ \\
\hline Allaitement maternel & 0,19 & $0,11-0,33$ & $<0,001$ \\
\hline Obésité $\left(\geq 30 \mathrm{~kg} / \mathrm{m}^{2}\right)$ & 2,76 & $1,63-4,71$ & $<0,001$ \\
\hline
\end{tabular}

Les données quantitatives sont exprimées en moyennes avec une déviation standard (DS) à 95\%. Les tests de comparaison ont été effectués à l'aide du X2 avec calcul des Odds Ratio (OR) et leurs intervalles de confiance (IC) à $95 \%$. Les différences ont été considérées comme statistiquement significatives au risque de $p<0,05$.

\section{Résultats}

L'âge moyen des patientes est de 51,1+14,2 ans, et similaire à celui des témoins, $50,7+14,4$ ans. Deux tiers des patientes $(63,3 \%)$ sont âgées entre 40 et 70 ans. Les femmes aux âges extrêmes sont représentées essentiellement par les plus jeunes (âge < 40 ans) avec $22,4 \%$ des cas et les femmes de plus de 70 ans représentent 14 , $3 \%$ de l'échantillon. Dans les deux groupes, l'âge moyen à la ménarchie est de 13,4 ans. Cependant, on note que la fréquence des ménarchies précoces avant 11 ans est plus élevée chez les cas $(10,4 \%)$ vs $3,0 \%$ chez les témoins $(p<0,001)$.

Les femmes présentant un cancer du sein sont plus souvent ménopausées au moment du diagnostic, $62,4 \%$ vs $46,0 \%$ chez les témoins $(p<0,001)$. Elles sont ménopausées à un âge plus précoce $49,9+3,32$ ans vs $55,1+5,5$ ans chez les témoins $(p<0,001)$. On observe ainsi chez les cas une vie génitale d'une durée moyenne de 35,4+0,4 ans. La ménopause tardive ( $>55$ ans) est plus souvent observée chez les témoins $(66,8 \%)$ que chez les cas $(7,7 \%)(p<0,001)$.

La contraception orale est plus fréquente chez les témoins $(69,1 \%$ vs $38,0 \%$ chez les cas, $p<0,001)$. Au total, $8,7 \%$ des cas ont utilisé des contraceptifs oraux pendant une durée de 4 ans avant la première grossesse, et 5,9\% d'entre elles ont eu une utilisation continue pendant une durée supérieure à 10 ans.

L'âge à la première grossesse est plus tardif chez les cas $(21,3+1,3$ ans) par rapport aux témoins $(20,4+0,65$ ans) $(p<0,001)$. Chez les cas, le pic de fréquence de l'âge aux premières grossesses se situe entre 14 et 24 ans, alors qu'il est situé entre 20 et 24 ans chez les témoins. Cependant, la fréquence des grossesses après 35 ans est similaire dans les deux groupes $(13,8 \%$ chez les cas vs $16,9 \%$ chez les témoins, $p=0,35$ ).

La multiparité ( 5 enfants et plus) est similaire dans les deux groupes, $53,8 \%$ chez les cas et $45,4 \%$ chez les témoins $(p=0,20)$. Les femmes atteintes de cancer du sein sont plus fréquemment nullipares $(13,2 \%$ vs $2,6 \%$ chez les cas, $p<0,001)$. Le taux d'avortement avant 3 mois est similaire dans les deux groupes $(31,0 \%$ chez les cas vs $32,8 \%$ chez les témoins).

Tableau 3 : Analyse stratifiée des facteurs de risque de cancer du sein selon les tranches d'âge

\begin{tabular}{|l|c|c|c|c|c|c|c|c|c|}
\hline \multicolumn{1}{|c|}{ Age } & \multicolumn{3}{|c|}{$<40$ ans } & \multicolumn{3}{c|}{$1-69 \quad$ ans } & \multicolumn{3}{c|}{$\leq 70$ ans } \\
\hline & OR & IC à $95 \%$ & $p$ & OR & IC à $95 \%$ & $p$ & OR & IC à $95 \%$ & $p$ \\
\hline Ménarchie précoce & 3,16 & $0,17-26,17$ & NS & 5,44 & $1,65-19,75$ & $<0,001$ & 1,09 & $0,10-12,06$ & NS \\
\hline Ménopause tardive & & & & 0,02 & $0,01-0,05$ & $<0,001$ & 0,01 & $0,00-0,05$ & NS \\
\hline Nulliparité & 14,4 & $4,76-45,75$ & $<0,001$ & 12,93 & $1,67-273,52$ & 0,002 & & & \\
\hline $\begin{array}{l}\text { Age tardif de la 1ère } \\
\text { grossesse }\end{array}$ & & & & 0,92 & $0,12-8,09$ & NS & & & \\
\hline Obésité & 0,26 & $0,11-0,60$ & 0,001 & 0,66 & $0,36-1,21$ & NS & 1,48 & $1,12-1,68$ & $<0,001$ \\
\hline
\end{tabular}


L'allaitement maternel est plus fréquemment retrouvé chez les témoins $(91,7 \%)$ que chez les cas $(67,6 \%)$ $(p<0,001)$. Il est à noter que la durée moyenne de l'allaitement chez les cas est de 8+0,3 mois.

Globalement, l'obésité se répartit équitablement dans les 2 groupes et concerne $31,7 \%$ des femmes. Cependant, si on examine la répartition de l'obésité selon les tranches d'âge, on s'aperçoit que dans le groupe des 70 ans et plus, celle-ci est significativement plus importante chez les cas $(12,5 \%)$ que chez les témoins $(3,5 \%)(p<0,001)$. La notion de mastopathie a été retrouvée chez $17,6 \%$ des cas.

L'étude des facteurs de risque dans l'ensemble de l'échantillon a montré une relation hautement significative du cancer du sein avec la ménarchie précoce $(\mathrm{OR}=3,76$, IC[1,55-9,41]), la nulliparité (OR=5,67, IC[2,30-14,62]), et l'obésité (OR=2,76, IC[1,63-4,71]) (tableau 2).

D'un autre côté, la ménopause tardive $(\mathrm{OR}=0,12, \mathrm{IC}[0,05$ $0,24])$, la multiparité ( 5 enfants et plus) $(O R=0,31$, IC $[0,21-0,47])$, la prise de contraceptifs $(O R=0,26$, IC $[0,18$ $0,41])$ et l'allaitement maternel $(O R=0,19, I C[0,11-0,33])$ apparaissent comme des facteurs hautement protecteurs $(p<0,001)$. Mais, en effectuant une stratification sur l'âge, on retrouve une très forte association entre le cancer du sein et la nulliparité chez les moins de 40 ans $(O R=14,4$, IC $[4,67-45,75])$ et dans le groupe des 40-69 ans (OR=12,93, IC $[1,67-273,52])$. Chez les 40-69 ans, la ménarchie précoce est fortement déterminante $(O R=5,44, I C[1,65-19,75])$,

alors que chez les femmes de 70 ans et plus, le cancer du sein est déterminé par l'obésité $(O R=1,48, I C[1,12-1,68])$.

\section{Discussion}

Toute proposition d'une politique de prévention primaire de cancer du sein doit s'appuyer sur une analyse approfondie des facteurs de risque actuellement identifiés [15].

Outre les facteurs de risque génétique ou biologique, certains comportements ou habitudes de vie peuvent augmenter le risque de cancer du sein [14]. Peu d'informations sont disponibles sur l'évolution de la prévalence des facteurs de risque chez la femme jeune. L'âge tardif de la première grossesse [16] et l'accroissement de l'obésité $[10,14,17]$ au cours de ces dernières années sont les facteurs, aux effets opposés, les mieux documentés.

L'âge est un facteur de risque dans la survenue du cancer du sein [1] même s'il peut atteindre des femmes à des âges très différents. Dans les populations européennes, le pic de fréquence se situe après 50 ans [17], alors que dans notre série, le pic de fréquence est observé plus tôt, entre 40 et 49 ans (30,5\% des cas). Ces différences sont probablement liées au patrimoine génétique de notre population, à la vie génitale de nos femmes, à leur mode alimentaire et à l'obésité fréquente qui touche une femme sur 3 [7]. Nous assistons en Algérie ces dernières années, à une recrudescence du cancer du sein avant 40 ans, pendant que la population générale subit un vieillissement qui explique cet excès d'incidence [5].

Dans notre série de cas, la fréquence des femmes qui ont connu une puberté précoce avant 11 ans, est de 10,4\%. Cette association avec cancer du sein est retrouvée chez les moins de 40 ans et les 40-69 ans (tableau 3).

De nombreuses études évoquent cette forte association entre la ménarchie précoce et le cancer du sein [18]. Le fondement biologique correspond à l'exposition précoce et prolongée à l'imprégnation hormonale qui existe durant la période d'activité des ovaires [19]. Les données suggèrent différents mécanismes biologiques, en particulier le rôle des hormones endogènes [18, 19].

On observe aussi que la fréquence des femmes ménopausées est plus élevée chez les cas que chez les témoins $(62,4 \%$ vs $46,0 \%, p<0,001)$ alors que la ménopause tardive enregistre des résultats contraires $(7,7 \%$ vs $66,8 \%, p<0,001)$. Ceci suggère que les hormones jouent un effet protecteur dans notre série, particulièrement dans la tranche d'âge 40-69 ans $(O R=0,001, I C[0,01-0,05], p<0,001)$. Ces résultats sont contraires aux données de la littérature qui estiment que la ménopause tardive constitue un facteur de risque [18]. L'influence de l'imprégnation hormonale à base d'œstrogènes et progestérone à laquelle est soumise une femme au cours de sa vie génitale, augmente le risque quand la ménopause survient après 55 ans [18]. Ce risque augmente d'environ 3\% pour chaque année supplémentaire d'activité génitale [20]. Ainsi, en raison du risque reconnu de l'œstradiol comme promoteur de la carcinogenèse, les diverses stratégies proposées pour la prévention du cancer du sein consistent toutes à diminuer la sécrétion d'œstradiol et à s'opposer à son action au niveau mammaire [18]. Les différences observées avec notre travail sont certainement liées à la taille réduite de notre échantillon.

D'un autre côté, nos résultats sur la durée de vie génitale, plus longue chez les cas $(35,4 \square 0,4$ ans) que chez les témoins ( $25,4 \square 4,9$ ans) sont en conformité avec les données de la littérature [21]. Ceux-ci rapportent que le risque de cancer du sein est lié à l'imprégnation hormonale observée au cours de cette période [21]. Ainsi dans notre série, $31,3 \%$ des cas présentent un délai ménarchie - première grossesse $>12$ ans vs $14,3 \%$ chez les témoins $(p<0,001)$.

La grossesse à terme avant 30 ans est considérée comme un facteur protecteur [22]. Un âge tardif à la première grossesse, après 35 ans, est plus favorable à l'apparition 
d'un cancer du sein, en raison des modifications hormonales gravidiques qui favorisent le développement des cellules moins différenciées, donc plus sensibles à une transformation néoplasique [23, 24]. Dans notre série, aucune relation significative n'a été retrouvée pour cette variable.

Il est reconnu par ailleurs que la multiparité a pour avantage de protéger les femmes contre le cancer du sein [25]. Plus une femme a des grossesses, moins elle est exposée à l'œstrogène circulant, contrairement aux femmes n'ayant porté aucun enfant ou n'ayant mené aucune grossesse à terme [26]. La multiparité n'apparait pas dans notre travail comme un facteur de risque compte tenu de sa fréquence élevée chez les femmes algériennes. Afin d'identifier cette variable en tant que facteur de risque, il est nécessaire de disposer d'un grand échantillon.

D'un autre côté, la nulliparité apparait aussi comme un facteur de risque dans notre échantillon $(O R=5,67$, $p<0,001$ ) à l'inverse des populations européennes chez qui ce facteur est plutôt protecteur [26]. Les facteurs d'exposition au risque hormonal endogène du cancer du sein contribuant à augmenter la prolifération cellulaire dans notre population, semblent plutôt liés à la non différenciation ou différenciation retardée de l'Unité Terminale Ductulo-Lobulaire (UTDL), qui sont rencontrés chez la nullipare et la première grossesse tardive [27].

Dans notre échantillon, la nulliparité qui se caractérise par une non différenciation de l'UTDL, est fortement associée au cancer du sein chez les moins de 70 ans.

La prise de contraceptifs oraux semble avoir un effet protecteur chez les témoins $(O R=0,26, p<0,001)$. A l'inverse, le risque lié à l'utilisation des contraceptifs oraux pendant une durée continue de 10 ans et plus, concerne près de $25 \%$ des porteuses de cancer du sein en Europe [15, 28]. De plus, dans ce contexte, l'âge de début de la prise de contraceptifs est très précoce. Dans notre échantillon, seulement $5,9 \%$ des cas ont présenté une durée de consommation de plus de 10 ans, avec un âge de début survenant après 20 ans pour $91 \%$ des cas. On observe aussi que cette consommation se fait de façon discontinue et anarchique. Ce mode d'utilisation mal documenté ne nous permet pas de mettre en évidence l'association cancer du sein et contraceptifs oraux. La relation cancer du seincontraceptifs oraux devrait être mieux documentée en raison des nombreux programmes de contraception orale mis en place depuis les années 80 et mal contrôlés.

L'allaitement apparait aussi dans notre série comme un facteur protecteur $(O R=0,19, p<0,001)$. Il est reconnu que l'effet protecteur de l'allaitement est lié en particulier à la diminution de la sécrétion des œstrogènes pendant la lactation et l'absence de cycles ovulatoires [16]. Cet effet protecteur est explicite après au moins 3 années d'allaitement [29].

D'autre part, l'obésité, elle-même liée à la sédentarité, est un facteur de risque important [10]. Elle est associée à un profil hormonal, en raison des concentrations sériques d'œstradiol libre, soupçonné de favoriser le développement du cancer du sein [30]. Le surpoids intervient dans la survenue du cancer du sein, en relation avec le tissu graisseux qui stocke facilement certaines hormones impliquées dans le développement de ces cancers [10, 30]. Dans notre échantillon, l'obésité n'apparait comme facteur de risque que chez les femmes de 70 ans et plus. Il est connu que ce risque est augmenté chez la femme ménopausée où le seul apport hormonal est apporté par les graisses stockées [30]. Dans les années à venir, le risque de cancer du sein lié à l'obésité est appelé à augmenter au vu de la croissance du surpoids dans notre population [7].

Il est à souligner que les principales limites de cette étude sont la taille réduite de l'échantillon et la non exhaustivité des facteurs de risque étudiés.

\section{Conclusion}

Cette étude a permis d'identifier la ménarchie précoce, la nulliparité et l'obésité comme facteurs de risque du cancer du sein chez la femme dans l'Ouest Algérien. Une identification de facteur de risque sur lesquels il est possible d'agir et une meilleure connaissance des mécanismes biologiques en cause, devraient faciliter la mise en œuvre de stratégie efficace de prévention dans cette maladie multifactorielle. Les effets des contraceptifs oraux sur l'incidence du cancer du sein restent un champ à explorer dans notre contexte suite à la multiplication des programmes dans le cadre du planning familial initié dès les années 80 .

La prévention primaire qui a pour but d'agir sur les facteurs modifiables tels que l'obésité [31], devrait s'appuyer sur des programmes sociaux et d'éducation pour la santé, afin de promouvoir chez les femmes, une alimentation équilibrée associée à une activité physique régulière.

Les directives recommandent d'individualiser les décisions de dépistage, en particulier chez les femmes de moins de 40 ans [32]. Ces recommandations se projettent vers une systématisation du dépistage par les tests génétiques [33]. Par ailleurs, une meilleure compréhension des mécanismes de l'oncogenèse spécifique permettra d'envisager des moyens de prévention du cancer du sein dans le cas de la nulliparité et des grossesses tardives.

La formation des personnels de santé et la sensibilisation 
des femmes à l'auto-palpation restent les meilleurs garants pour un diagnostic précoce susceptible d'améliorer la guérison.

\section{Conflits d'intérêts}

Les auteurs déclarent n'avoir aucun conflit d'intérêt en rapport avec cette étude.

\section{Remerciements}

Nos remerciements s'adressent au Professeur Midoun, EHU-Oran, pour son aide, ainsi qu'à toutes les patientes et les témoins pour leur précieuse collaboration.

\section{Références bibliographiques}

[1] IARC Cancer Base International Agency for Research on Cancer. Cancer incidence and mortality worldwide. 2012

[2] WHO. The global burden of disease: 2004 update. 2008

[3] Coleman MP1, Quaresma M, Berrino F, Lutz JM, De Angelis R, CONCORD Working Group. Cancer survival in five continents: a worIdwide population-based study (CONCORD). Lancet Oncol 2008, 9: 730-56.

[4] Ministère de la Santé de la Population et de la Réforme Hospitalière, Algérie. Plan National Cancer. Octobre 2014

[5] Hamdi Cherif Mokhtar, Mahnane Abbes, Laouamri Slimane, Zaidi Zoubida, Boukharouba Hafida, Cherka Dahbia, Rakeb Manel, Kara Lamia, Ayat. Cancer à Sétif 1986-2010, Algérie : Incidences, Tendances et Survie. Registre du Cancer de Sétif. Novembre 2013.

[6] Anderson BO, Yip CH, Smith RA, Shyyan R, Sener SF, Eniu A, Carlson RW, Azavedo E, Harford J. Guideline implementation for breast healthcare in low-income and middle-income countries: overview of the Breast Health Global Initiative Global Summit 2007. Cancer 2008, 113: 2221-43.

[7] Institut National de Santé Publique. Transition épidémiologique et système de santé. Projet TAHINA Résultats Enquête Nationale Santé. 2007, [http://www.ands.dz/insp/DOC_ENS_Novembre_2007_tahina.pdf].

[8] Danaei G, Vander Hoorn S, Lopez AD, Murray CJ, Ezzati M; Comparative Risk Assessment collaborating group (Cancers). Causes of cancer in the world: comparative risk assessment of nine behavioural and environmental risk factors. Lancet 2005, 366: 1784-93.

[9] Kispert S, McHowat J. Recent insights into cigarette smoking as a lifestyle risk factor for breast cancer. Breast Cancer (Dove Med Press) 2017. 7(9): 127-132. doi: 10.2147/BCTT.S129746. eCollection 2017.

[10] Renehan AG, Tyson M, Egger M, Heller RF, Zwahlen M. Body-mass index and incidence of cancer: a systematic review and meta-analysis of prospective observational studies. The Lancet 2008. 371(9612): 569-578

[11] Calle EE, Rodriguez C, Walker-Thurmond K, Thun MJ. Overweight, obesity, and mortality from cancer in a prospectively studied cohort of U.S. adults. N Engl J Med 2003. 348: 1625-38.

[12] Eliassen AH, Hankinson SE, Rosner B, Holmes MD, Willett WC. Physical Activity and Risk of Breast Cancer Among Postmenopausal Women. Arch Intern Med 2010, 170 (19): 1758-1764.doi:10.1001/ archinternmed.2010.363

[13] IARC. World cancer report 2008. Lyon, International Agency for Research on Cancer 2008.

[14] Lacey JV Jr, Kreimer AR, Buys SS, Marcus PM, Chang SC, Leitzmann MF, Hoover RN, Prorok PC, Berg CD, Hartge P; Prostate, Lung, Colorectal and Ovarian (PLCO) Cancer Screening Trial Project Team. Breast cancer epidemiology according to recognized breast cancer risk factors in the Prostate, Lung, Colorectal and Ovarian (PLCO) Cancer Screening Trial Cohort. BMC Cancer 2009, 9: 84.

[15] Haute Autorité de Santé. Dépistage du cancer du sein en France : identification des femmes à haut risque et modalités de dépistage. Mars 2014

[16] Andrieu N, Goldgar DE, Easton DF, Rookus M, Brohet R, Antoniou AC. Pregnancies, Breast-Feeding, and Breast Cancer Risk in the International BRCA1/2 Carrier Cohort Study (IBCCS). J. Natl. Cancer Inst. 2006, 98(8): 535- 544

[17] Kelsey JL, Beinstein L. Epidémiology and prevention of breast cancer Ann Rev pub Health 1996, 17: 47- 67

[18] Collaborative Group on Hormonal Factors in Breast Cancer. Menarche, meno-pause, and breast cancer risk: individual participant meta-analysis, including 118964 women with breast cancer from 117 epidemiological studies. Lancet Oncol 2012, 13:1141-51. doi:10.1016/S1470-2045(12)70425-4

[19] Rosenberg PS, Barker KA, Anderson WF. Estrogen receptor status and the future burden of invasive and in situ breast cancers in the United States. J Natl Cancer Inst 2015, 107:djv159. doi:10.1093/jnci/djv159

[20] Collaborative Group on Hormonal Factors in Breast Cancer. Breast cancer and hormone replacement therapy: collaborative reanalysis of data from 51 epidemiological studies of 52705 women with breast cancer and 108411 women without breast cancer. Lancet 1997, 350:1047-59. doi:10.1016/ S0140-6736(97)08233-0 12.

[21] Clavel-Chapelon, F. Cumulative number of menstrual cycles and breast cancer risk:results from the E3N cohort study of French women. Cancer Causes and Control 2002, 13(9): 831-838

[22] Lesieur B, Vercambre M, Dubernard G, Khosrotehrani K, Uzan $\mathrm{S}$, Aractingi $\mathrm{S}$, Rouzier R Risk breast cancer related of pregnancy. J Gynecol Obstet Biol Reprod (Paris) 2008, 37(1):77-81. Epub 2007 Dec 20

[23] Albrektsen G, Heuch I, Hansen S, Kvale G. Breast cancer risk by age at birth, time since birth and time intervals between births: exploring inter-action effects. Br J Cancer 2005, 92:167-75. doi:10.1038/sj.bjc. 6602302

[24] Ma H, Henderson KD, Sullivan-Halley J, Duan L, Marshall SF, Ursin $G$, et al. Pregnancy-related factors and the risk of breast carcinoma in situ and inva-sive breast cancer among postmenopausal women in the California Teachers Study cohort. Breast Cancer Res 2010, 12:R35. doi:10.1186/bcr2589

[25] Hinkula M, Pukkala E, Kyyronenp, Kauppila A grand multiparity and the risk of breast cancer: population based study in finland. Cancer Causes Control 2001, 12: 491-500

[26] Ma H, Bernstein L, Pike MC, Ursin G. Reproductive factors and 
breast cancer risk according to joint estrogen and progesterone receptor status: a meta-analysis of epidemiological studies. Breast Cancer Res 2006, 8:R43. doi:10.1186/bcr1525

[27] M Espié, A Gorins. Le sein. Edition Eska 2007

[28] Althuis MD, Brogan DR, Coates RJ, Daling JR, Gammon MD, Malone KE. Hormonal content and potency of oral contraceptives and breast cancer risk among young women. Br J Cancer 2003, 88: 50-7. doi:10.1038/sj. bjc.6600691

[29] Key TJ, Verkasolo PK, Banks E. Epidemiology of breast cancer. Lancet oncology 2001, 2(3): 133-40

[30] Wenten M1, Gilliland FD, Baumgartner K, Samet JM. Associations of weight, weight change, and body mass with breast can- cer risk in Hispanic and non-Hispanic white women. Ann Epidemio 2002, 12(6): 435-41

[31] Rochefort H1, Rouëssé J, Groupe de Travail de la Commission III (Cancérologie). Comment réduire l'incidence du cancer du sein. Bull Acad Natl Med. 2008, 192(1): 161-79

[32] WHO. Cancer control: knowledge into action: WHO guide for effective programmes: early detection. 2007

[33] Pace L, Keating NL. A systematic assement of benefits and risk to guide breast cancer screnning decisions. JAMA 2014, 311: 1327-1335 